\title{
Astaxanthin in microalgae: pathways, functions and biotechnological implications
}

\author{
Danxiang $\mathrm{Han}^{1}$, Yantao $\mathrm{Li}^{2}$ and Qiang $\mathrm{Hu}^{\mathrm{1}, *}$ \\ ${ }^{1}$ Laboratory for Algae Research and Biotechnology, College of Technology and Innovation, Arizona State University Poly- \\ technic Campus, Mesa, AZ 85212, USA \\ ${ }^{2}$ The Institute of Marine and Environmental Technology (IMET), The University of Maryland, Baltimore, MD 21202, USA
}

\begin{abstract}
Major progress has been made in the past decade towards understanding of the biosynthesis of red carotenoid astaxanthin and its roles in stress response while exploiting microalgae-based astaxanthin as a potent antioxidant for human health and as a coloring agent for aquaculture applications. In this review, astaxanthin-producing green microalgae are briefly summarized with Haematococcus pluvialis and Chlorella zofingiensis recognized to be the most popular astaxanthin-producers. Two distinct pathways for astaxanthin synthesis along with associated cellular, physiological, and biochemical changes are elucidated using H. pluvialis and C. zofingiensis as the model systems. Interactions between astaxanthin biosynthesis and photosynthesis, fatty acid biosynthesis and enzymatic defense systems are described in the context of multiple lines of defense mechanisms working in concert against photooxidative stress. Major pros and cons of mass cultivation of H. pluvialis and C. zofingiensis in phototrophic, heterotrophic, and mixotrophic culture modes are analyzed. Recent progress in genetic engineering of plants and microalgae for astaxanthin production is presented. Future advancement in microalgal astaxanthin research will depend largely on genome sequencing of H. pluvialis and C. zofingiensis and genetic toolbox development. Continuous effort along the heterotrophic-phototrophic culture mode could lead to major expansion of the microalgal astaxanthin industry.
\end{abstract}

Key Words: astaxanthin biosynthesis; Chlorella zofingiensis; genetic engineering; Haematococcus pluvialis; mass culture; photooxidative stress

\section{INTRODUCTION}

Astaxanthin (3,3'-dihydroxy- $\beta, \beta$-carotene- $4,4^{\prime}$-dione) is a ketocarotenoid synthesized by limited numbers of microalgae, plants, bacteria, and fungi. In microalgae, unlike primary carotenoids (e.g., $\beta$-carotene, zeaxanthin, and lutein) which constitute structural and functional components of the photosynthetic apparatus, astaxanthin is a secondary carotenoid accumulating in cytosolic lipid bodies (LBs) only under environmental stress or adverse culture conditions, such as high light, high salinity, and nutrient deprivation (Johnson and An 1991, Johnson and Schroeder 1996).

Astaxanthin has thirteen conjugated double bonds arranged as alternating single-double bonds. This configuration confers astaxanthin strong antioxidant properties thereby scavenging reactive oxygen species (ROS) and neutralizing free radicals (Miki 1991, Vershinin 1999). Because of this, astaxanthin has been used as a nutraceutical and a pharmaceutical, for example, to fight against (c) This is an Open Access article distributed under the terms of the Creative Commons Attribution Non-Commercial License (http://creativecommons.org/licenses/by-nc/3.0/) which permits unrestricted non-commercial use, distribution, and reproduction in any medium, provided the original work is properly cited.
Received April 15, 2013, Accepted May 21, 2013

* Corresponding Author

E-mail: huqiang@asu.edu

Tel: +1-480-727-1484, Fax: +1-480-727-1236 
free-radical associated diseases like oral, colon and liver cancers, cardiovascular diseases, and degenerative eye diseases (Lorenz and Cysewski 2000, Guerin et al. 2003). Astaxanthin is also a common coloring agent in aquaculture to impart red pigmentation in animal bodies such as salmon and rainbow trout (Lorenz and Cysewski 2000, Guerin et al. 2003).

The current world astaxanthin market is dominated by synthetic astaxanthin. The price for synthetic astaxanthin is above $\$ 2,000 \mathrm{~kg}^{-1}$, and the total market value of astaxanthin is over $\$ 240 \mathrm{M}$ per year (Misawa 2009, Li et al. 2011). Synthetic astaxanthin typically contains a mixture of $3 S$, 3 'S; $3 R, 3$ 'S; and $3 R, 3^{\prime} R$ isoforms with a ratio of $1: 2: 1$. In contrast, astaxanthin from microalgae is predominantly the $3 S$, 3'S isomer. The $3 S, 3$ 'S astaxanthin isomer is a preferable form as a feed additive in aquaculture because it imparts a higher extent of pigmentation in rainbow trout than the synthetic astaxanthin (Barbosa et al. 1999). There are growing concerns about the safety of using synthetic astaxanthin for aquaculture or direct human consumption and therefore natural astaxanthin represents a preferred and premium choice for human consumption (Li et al. 2011).

Astaxanthin can be produced in various amounts by a number of microalgae including Botryococcus braunii (up to $0.01 \%$ astaxanthin by dry weight [dwt]) (Grung et al. 1994), Chlamydocapsa spp. (0.04\% astaxanthin by dwt) (Leya et al. 2009), Chlamydomonas nivalis (Bidigare et al. 1993, Remias et al. 2005), Chlorella zofingiensis (0.7\% astaxanthin by dwt) (Bar et al. 1995, Orosa et al. 2000), Chlorococcum sp. (0.7\% astaxanthin by dwt) (Liu and Lee 2000, Ma and Chen 2001), Chloromonas nivalis $(0.004 \%$ astaxanthin by dwt) (Leya et al. 2009, Remias et al. 2010), Eremosphera viridis (Vechtel et al. 1992), Haematococcus pluvialis (4\% astaxanthin by dwt) (Droop 1954, Lee and Ding 1994), Neochloris wimmeri (1.9\% astaxanthin by dwt) (Orosa et al. 2000), Protosiphon botryoides (1.4\% astaxanthin by dwt) (Orosa et al. 2000), Scenedesmus sp. (0.3\% astaxanthin by dwt) (Orosa et al. 2000, Qin et al. 2008), Scotiellopsis oocystiformis (1.1\% astaxanthin by dwt) (Orosa et al. 2000), and Trachelomonas volvocina (Green 1963). Of these species, the green microalga $H$. pluvialis is recognized as one of the most promising producer of astaxanthin in nature due to its exceptional ability to accumulate large amounts of astaxanthin under stress conditions (Boussiba 2000, Lemoine and Schoefs 2010). Recently, C. zofingiensis has attracted some interest as an alternative astaxanthin producer, due to its ability to grow rapidly (with a maximum specific growth rate of $1.03 \mathrm{~d}^{-1}$ and biomass concentration of $53 \mathrm{~g} \mathrm{~L}^{-1}$ when grown on glucose) and adopt a photoautotrophic, mixotrophic, or heterotropic culture mode (Orosa et al. 2000, Del Campo et al. 2004, Ip et al. 2004, Sun et al. 2008).

The biosynthesis of astaxanthin in microalgae is usually accompanied by the transformation of the algae from a green vegetative form into a red cyst (Droop 1954). The cellular and molecular responses of $H$. pluvialis and $C$. zofingiensis and to stress are proposed to result, at least in part, from differential expression of carotenoid synthesis genes at the transcriptional level (Hershkovits et al. 1997, Li et al. 2008a, 2010). Recently, many genes responsible for carotenoids, particularly astaxanthin biosynthesis have been cloned and characterized in H. pluvialis and C. zofingiensis, which has provided the opportunities to study the pathways and regulation of carotenoid biosynthesis and understand the biological role of astaxanthin in stress response. This review summarizes the cell biology, physiological, and biochemical characteristics of $H$. pluvialis and C. zofingiensis, with emphasis on recent progress on the pathways for and the physiological role of astaxanthin synthesis under stress. The biotechnological implications of these studies are discussed and recent efforts on genetic engineering of algal genes in microalgae and higher plants for overproduction of astaxanthin are described.

\section{HAEMATOCOCCUS PLUVIALIS}

\section{Cell biology and physiology}

The life cycle of $H$. pluvialis consists of four types of distinguishable cells, including macrozooids (or zoospores), microzooids, palmella, and aplanospores (or haematocysts) (Elliot 1934). Macrozooids are spherical, ellipsoidal, or pear-shaped cells with two flagella and a cup-shaped chloroplast. Exhibited by rapid growth and cell division producing 2-8 daughter cells, macrozooids predominate in the early vegetative growing stage of a batch culture under favorable culture conditions. When macrozooids are subjected to unfavorable environmental or culture conditions, they develop into a non-motile 'palmella' form by losing their flagella while expanding the cell size. This process is defined as encystment. When the stress persists, 'palmella' will develop into the non-motile asexual aplanospores with thick and rigid cell walls. During the maturation of aplanospores, large amounts of astaxanthin accumulate, which brings a bright red color to the cells. It is worth noting that macrozooids of some $H$. pluvialis strains are capable of accumulating astaxan- 
thin without forming alpanospores (Brinda et al. 2004, Hagen et al. 2004). If the environmental or culture conditions are back to normal, aplanospores will germinate to form flagellated zoospores to start a new vegetative growth cycle. In some cases, after exposure to extreme adverse conditions, e.g., freezing, desiccation, or nutrient starvation, followed by favorable culture conditions, gametogenesis may occur in aplanospores, giving rise to up to 64 gametes, known as microzooids. The microzooids appear as smaller cells $(<10 \mu \mathrm{m})$ as compared to the zoospores $(20-50 \mu \mathrm{m})$, and exhibit high-speed motility after their release from gametocysts (Triki et al. 1997).

Accumulation of astaxanthin in H. pluvialis cells is accompanied by profound ultrastrutral changes. One of the most remarkable changes is the formation of a large number of cytosolic LBs where bulks of astaxanthin molecules are stored. Initially, LBs appear as tiny electrondense spots scattered near the rough endoplasmic reticulum cisternae and eventually are coalesced to form large mature LBs (Lang 1968, Santos and Mesquita 1984).

Flagellated zoospores of $H$. pluvialis exhibit a voluminous extracellular matrix, resembling the typical Vovocalean multilayered architecture with a tripartite crystalline layer between two separate layers of gelatinous matrices. The tripartite crystalline layer is connected to the cytoplasma membrane by fine radiating strands, which is only observed in the genus of Haematococcus. Encystment of the flagellated zoospores into non-motile palmella cells is characterized by the formation of the primary cell wall within the extracellular matrix (Hagen et al. 2008). The primary cell wall can be stained with calcofluor white, indicative of the presence of $\beta$-1,4-glycosidic linkages. Further formation of red aplanospores is concurrent with the development of secondary cell wall, which contains $3 \%$ acetolysis-resistant materials suggested to be algaenan, an aliphatic biopolymer with great resistance to chemicals (Damiani et al. 2006, Hagen et al. 2008). Recently, the proteomes of $H$. pluvialis cell wall at different developmental stages were characterized, suggesting possible involvement of cellulose synthesis in the primary cell formation (Wang et al. 2004b).

The most noticeable response of $H$. pluvialis to adverse culture conditions is to synthesize and accumulate large amounts of astaxanthin in the form of mono- and di-esters. While the initial astaxanthin synthesis utilizes the existing $\beta$-carotene as a precursor, produce a bulk of astaxanthin esters will depend on de novo $\beta$-carotene synthesis (Schoefs et al. 2001).

Nearly $50 \%$ reduction in chlorophyll content occurred during the transformation of green vegetative cells into red aplanospores (Boussiba et al. 1999, Han et al. 2012), and yet red aplanospores were photosynthetically active with only moderate declines in the maximal photosynthetic rate $\left(P_{\max }\right)$ and the maximum quantum yield of photosystem II (PSII), presumably attributable to the relatively stable photosynthetic unit size and the activated D1 repairing mechanism (Zlotnik et al. 1993, Wang et al. 2003, Qiu and Li 2006). Attenuated linear electron transport as indicated by significantly reduced cytochrombe $b 6 f$ content and the concomitant enhanced plastid terminal oxidase (PTOX)-mediated alternative electron transport were considered to prevent the photosynthetic electron transport chain from being over-reduced when H. pluvialis cells are under photooxidative stress (Tan et al. 1995, Han et al. 2012). On the other hand, the PSI activity was significantly enhanced during the transformation of green vegetative cells to red aplanospores (Hagen et al. 1993, Tan et al. 1995, Qiu and Li 2006).

\section{CHLORELLA ZOFINGIENSIS}

The unicellular microalgae Chlorella spp. have been widely used in basic biology research and various fields of biotechnology, such as animal feeds, health food and bioenergy production (Huss et al. 1999). C. zofingiensis (Chlorellaceae) has a rigid cell wall containing glucose and mannose, and is able to accumulate secondary carotenoids, whereas other species of Chlorella, such as $C$. vulgaris, have a glucosamine-containing cell wall and no capability of accumulating secondary carotenoids (Takeda 1991).

In response to stress, C. zofingiensis accumulates astaxanthin and canthaxanthin in the cytosolic LBs with concomitant degradation of chloroplast membranes (Rise et al. 1994, Bar et al. 1995). Degradation of thylakoids can be observed within $4 \mathrm{~h}$ when C. zofingiensis was exposed to high light and nitrogen deprivation, and the grana structure of the thylakoids disappeared with drastic decrease of thylakoid membranes after $24 \mathrm{~h}$ under stress. After 3 days, the chloroplast of $C$. zofingiensis lost its typical membrane structure (Bar et al. 1995). A thin lipid-rich layer containing the ketocarotenoids was observed at day 3 , possibly function as a sunshade filter to reduce light penetration into the chloroplast and thus prevent formation of excess ROS, a mechanism also found in H. pluvialis (Hagen et al. 1994, Bar et al. 1995). Like H. pluvialis, a very thick cell wall was observed in $C$. zofingiensis cells under stress, which was resistant to concentrated sulfuric acid treatment. The close proximity of the ketocarot- 
enoids layer to the cell wall suggested that the secondary carotenoids may be used as substrates for synthesis of sporopollenin in the cell walls (Bar et al. 1995).

The secondary carotenoids accumulated in C. zofingiensis under stress are canthaxanthin and astaxanthin in the forms of free, monoesters and diesters of the pigments (Rise et al. 1994). Canthaxanthin, free astaxanthin and monoesters were detected within $4 \mathrm{~h}$ flowing nitrogen deprivation and high light stress, while astaxanthin diesters appeared at $12 \mathrm{~h}$. After 3 days, the main secondary carotenoids accumulated in the cell were canthaxanthin (ca. 25\%) and astaxanthin monoesters (ca. 50\%) (Bar et al. 1995). Interestingly, C. zofingiensis grown heterotrophically can also accumulate significant amounts of astaxanthin (Ip and Chen 2005b). A survey of different carbon sources showed that glucose, mannose, fructose, sucrose, galactose and lactose can be used by C. zofingiensis, of which glucose and mannose were the best carbon sources for growth and astaxanthin biosynthesis in the dark. A high astaxanthin yield of $32 \mathrm{mg} \mathrm{L}^{-1}$ was achieved in a feedbatch culture of this organism (Sun et al. 2008).

\section{ASTAXANTHIN BIOSYNTHESIS PATHWAYS}

\section{Biosynthesis of astaxanthin in Haematococcus pluvialis}

Isopentenyl pyrophosphate (IPP) is the precursor for carotenoid synthesis (Lichtenthaler 1999). Two distinct pathways for IPP biosynthesis have been found in higher plants: the mevalonate pathway in the cytosol and the non-mevalonate 1-deoxy-D-xylulose-5-phosphate pathway in the chloroplast (DOXP pathway or MEP pathway) (Lichtenthaler et al. 1997). In unicellular green microalgae H. pluvialis and Chlamydomonas reinhardtii, IPP is believed to be synthesized solely from the non-mevalonate DOXP pathway (Disch et al. 1998). Subsequently, the isopentenyl pyrophosphate isomerase (IPI) catalyzes the isomerization of IPP to dimethylallyl diphosphate (Lichtenthaler 1999). Introduction of a heterologous IPI gene in Escherichia coli enhanced the accumulation of carotenoids, supporting a role of IPI in regulating carotenoid biosynthesis (Kajiwara et al. 1997, Wang et al. 1999). Two cDNAs of IPI, IPI1 and IPI2 have been cloned and characterized in H. pluvialis (Sun et al. 1998). Transcripts of both IPI genes increased in response to oxidative stress, but only IPI2 was up-regulated at the translational level. Moreover, only IPI2 protein was detected in mature red cysts in which astaxanthin was accumulated, suggesting that IPI2 is responsible for synthesis of the secondary carotenoids, whereas the IPII is responsible for primary carotenoid synthesis in the chloroplast of $H$. pluvialis (Sun et al. 1998).

Phytoene synthase (PSY) catalyzes the first committed step for carotenoid biosynthesis through condensation of two 20-carbon geranylgeranyl pyrophosphate molecules to form a 40-carbon phytoene, the precursor for all other carotenoids (Cunningham and Gantt 1998). Two classes of PSY were found in certain green algae like Ostrecoccus and Micromonas, while some other green algae like $C$. reinhardtii and C. vulgaris only possess one class of PSY (Ttran et al. 2009a) One copy of PSY gene has been cloned and characterized from a number of microorganisms including H. pluvialis (Steinbrenner and Linden 2001) and C. zofingiensis (Cordero et al. 2011). The Haematococcus PSY has an N-terminal extension similar to a chloroplast targeting sequence, indicating that PSY is likely to be targeted to the chloroplast in $H$. pluvialis (Steinbrenner and Linden 2001).

Through a series of dehydrogenation reactions, two structurally similar enzymes, phytoene desaturase (PDS) and $\zeta$-carotene desaturase (ZDS) convert the colorless phytoene into red lycopene. Specifically, PDS catalyzes the first two dehydrogenation reactions to form phytofluene and $\zeta$-carotene, whereas ZDS catalyzes two further reactions converting $\zeta$-carotene to neurosporene and lycopene (Cunningham and Gantt 1998). These dehydrogenation reactions extend the conjugated carbon-carbon double bonds to form the chromophore of carotenoids. These FAD-containing enzymes require PTOX and plastoquinone (PQ) as electron acceptors (Carol et al. 1999, Wu et al. 1999). The PDS and two PTOX genes (i.e., PTOXI and PTOX2) have been cloned and characterized in $H$. pluvialis (Grunewald et al. 2000, Wang et al. 2009, Li et al. 2010). High light illumination and nitrogen deprivation increase the transcripts of $P D S$ and $P T O X$ simultaneously in H. pluvialis, suggesting that PDS and PTOX may act in concert to dehydrogenate phytoene and remove excess electrons under stress, thereby preventing over-reduction of the photosynthetic electron transport chain and the formation of excess ROS (Grunewald et al. 2000, Wang et al. 2009, Li et al. 2010). Western-blot and immunogold labeling experiments showed that PDS is located exclusively in the chloroplast in H. pluvialis (Grunewald et al. 2000).

The cyclization of lycopene catalyzed by lycopene $\beta$-cyclase (LCY-b) and lycopene $\varepsilon$-cyclase (LCY-e) is a branching point in the carotenoid biosynthesis (Cunningham and Gantt 1998). LCY-b catalyzes two $\beta$-cyclization 


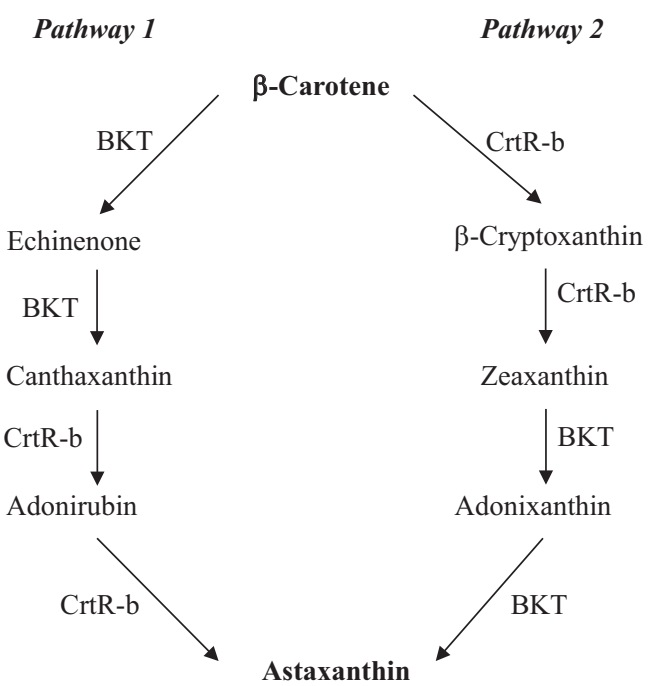

Fig. 1. Primary astaxanthin biosynthesis pathways in Haematococcus pluvialis (pathway 1) and Chlorella zofingiensis (pathway 2). Enzymes are named according to the designation of their genes. BKT, $\beta$-carotenoid ketolase; $C r t R-b, \beta$-carotenoid hydroxylase. Modified and reproduced from $\mathrm{Li}$ (2007) with permission from the University of Hong Kong.

reactions at each end of lycopene to form $\beta$-carotene, which is an end product for photosynthesis in plants and algae, as well as a precursor for keto-carotenoids in the chloroplast and cytosol. LCY-e catalyzes $\varepsilon$-cyclization of lycopene to $\zeta$-carotene, which is subsequently cyclized by LCY-b to form $\alpha$-carotene ( $\beta, \varepsilon$-carotene), a precursor for lutein synthesis (Cunningham et al. 1996). A LCY-b gene has been cloned in $H$. pluvialis and was observed that its transcripts increased concomitantly with the formation of $\beta$-carotene and astaxanthin at increased intensities of red or blue light (Steinbrenner and Linden 2003).

Oxygenation of $\beta$-carotene at the 4 position by $\beta$-carotene ketolase (BKT, synonym $\mathrm{CrtW}$ ) gives rise to echinenone and canthaxanthin (Fig. 1). In H. pluvialis, three different $B K T$ genes have been cloned, including BKT1 (Lotan and Hirschberg 1995), BKT2 (Kajiwara et al. 1995), and BKT3 (Huang et al. 2006a). Under stress, the multiple $B K T$ genes were up-regulated and when total $B K T$ mRNAs reached a certain threshold, $H$. pluvialis began to synthetize astaxanthin (Huang et al. 2006a). Immuno-localization study indicated that BKTs were localized in both the chloroplast and cytosolic LBs in $H$. pluvialis, although ketolase activity was detected only in the cytosolic LB preparation (Grünewald et al. 2001). Since PDS is an early enzyme in the pathway that is located exclusively in the chloroplast (Grunewald et al. 2000), it was proposed that transport of carotenoid precursors from the chloroplast to cytosolic LBs by a yet unknown mechanism would be necessary for astaxanthin formation (Grünewald et al. 2001).

Heterologously expressed Haematococcus BKT1 was not able to use dihydroxy carotenoid zeaxanthin as a substrate, indicating that the oxygenation steps likely precede hydroxylation steps (Lotan and Hirschberg 1995). In vitro assay of BKT and $\beta$-carotene hydroxylase (CrtRb, synonym Chy-b) further confirmed that astaxanthin synthesis in H. pluvialis is preferably preceded with the addition of keto groups, followed by the hydroxylation reactions (Fraser et al. 1998, Schoefs et al. 2001). Astaxanthin in $H$. pluvialis is proposed to be synthesized from the hydroxylation of canthaxanthin catalyzed by CrtR-b (Fig. 1). CrtR-b gene has been isolated in H. pluvialis (Linden 1999). A linear relationship between the increase of $C r t R-b$ transcripts and astaxanthin concentration was observed in the $H$. pluvialis wild type and the astaxanthin-hyper-accumulating mutant (MT 2877), suggesting a transcriptional control of $C r t R-b$ over astaxanthin synthesis (Li et al. 2008b). BKT and CrtR-b have been often proposed as rate-limiting steps in astaxanthin synthesis. Genetic manipulation of their genes in algae and plants for enhanced astaxanthin production is further discussed in the BIOTECHNOLOGICAL IMPLICATIONS section.

\section{Regulation of carotenogenesis in Haematococ- cus pluvialis}

Most Haematococcus carotenoid synthesis genes are up-regulated at the transcriptional level in response to various stressors (e.g., high light, excess ferrous sulfate and excess sodium acetate) (Li et al. 2008b). The extent to which the individual stressor affecting carotenogenesis gene expression follows the order as: low light $<$ low light + ferrous sulfate $<$ low light + sodium acetate $<$ high light + ferrous sulfate/sodium acetate $<$ high light + ferrous sulfate + sodium acetate ( $\mathrm{Li}$ et al. 2008b). Interestingly, when multiple factors were applied simultaneously to $H$. pluvialis, for example, high light + salt stress + iron stress, the transcripts of carotenoid synthesis genes were lower than and the maximum expression levels of those genes were delayed compared to that induced by the individual stressors applied separately or two stressors together at the early stage of stress (Li et al. 2008b). A plausible interpretation was that $H$. pluvialis and other microalgae possess both "house-keeping" defense mechanisms and stressor-specific defense mechanisms. For example, the carotenogenesis for production of astaxanthin and other secondary carotenoids is largely induced under high light stress, whereas various enzymatic defense reactions may 
be triggered by salinity or excess iron ion. When multiple stressors were applied to $H$. pluvialis, multiple stress protection mechanisms may all be activated and work in concert, each contributing partially to overall cell protection and to a less extent carotenogenesis. However, because the enzymatic defense systems mainly serve as short-team cellular defense mechanisms in $H$. pluvialis (Wang et al. 2004a), as the stress persists, the cells would have to depend more upon the long-term defense mechanism, -carotenogenesis, for survival, which explained why an delayed expression pattern of carotenogenesis genes with greater expression levels occurred in the cultures treated with the multiple stressors (Li et al. 2008b).

ROS play a role in regulation of carotenogenesis in $H$. pluvialis. Treatment with ROS-generating compounds such as methylviologen, methylene blue and $\mathrm{Fe}^{2+}$, increased PDS and CrtR- $b$ expression in H. pluvialis. They also induced astaxanthin synthesis independent of de novo protein synthesis (Kobayashi et al. 1993, Steinbrenner and Linden 2001), suggesting ROS mediate stress response and carotenogenesis in H. pluvialis at a post-translational level (Steinbrenner and Linden 2003). Unlike PDS and $C r t R-b$, however, treatment with various transcriptional and translational inhibitors suggested that $B K T$ gene expression was dependent on de novo protein synthesis (Vidhyavathi et al. 2008). Considering PDS is shown to be located in the chloroplast, whereas BKT activity is found in the cytosolic LBs, this discrepancy can be explained by the distinct subcellular localization of individual carotenoid biosynthetic enzymes.

\section{Pathways and regulation of carotenogenesis in Chlorella zofingiensis}

C. zofingiensis has the identical $\beta$-carotene synthesis pathway found in $H$. pluvialis, but may take a different route to make astaxanthin (Fig. 1) (Li 2007). Recently, the genes involved in biosynthesis of astaxanthin in $C$. zofingiensis have been cloned and characterized, including PSY (Cordero et al. 2011), PDS (Huang et al. 2008), $L C Y-b$ (Cordero et al. 2010), LCY-e (Cordero et al. 2012), $B K T$ (CrtO) (Huang et al. 2006b), CrtR-b (Chy-b) (Li et al. 2008a). Under high-light conditions, PSY, PDS, BKT, $C r t R-b$ genes were up-regulated, whereas the mRNA levels of $L C Y-b$ and $L C Y$-e remained constant, leading to formation of secondary carotenoids (Li et al. 2009, Cordero et al. 2012).

Functional analysis of C. zofingiensis BKT demonstrated that this enzyme not only converted $\beta$-carotene to canthaxanthin via echinenone, but also exhibited high enzymatic activity of converting zeaxanthin to astaxanthin via adonixanthin (Huang et al. 2006b). When the BKT inhibitor diphenylamine was applied, C. zofingiensis accumulated zeaxanthin while $H$. pluvialis accumulated $\beta$-carotene (Wang and Chen 2008), suggesting that $C$. zofingiensis synthesizes astaxanthin through zeaxanthin (Fig. 1, Pathway 2).

Various environmental factors may differentially regulate carotenogenesis in $C$. zofingiensis. High irradiance triggered up-regulation of PDS, BKT, CrtR- $b$ genes in $C$. zofingiensis, with most significant increase in $C r t R-b$ transcripts, leading to the accumulation of canthaxanthin, zeaxanthin and astaxanthin (Li et al. 2009). Under salt stress, only $B K T$ gene was up-regulated and canthaxanthin and astaxanthin were accumulated (Li et al. 2009). When fed with sugars, C. zofingiensis accumulated astaxanthin in dividing cells. Glucose and mannose were the best carbon sources to sustain growth and astaxanthin production in C. zofingiensis, followed by fructose, sucrose and galactose, whereas lactose was the poorest carbon source for C. zofingiensis (Sun et al. 2008). In heterotrophically grown C. zofingiensis, carotenogenesis was regulated through the phosphorylation of glucose by hexokinase, which was essential for up-regulation of $C r t R-b$ and synthesis of zeaxanthin, whereas the signals from the mitochondrial alternative pathway may mediate up-regulation of $B K T$ and synthesis of astaxanthin ( $\mathrm{Li}$ et al. 2008a).

Two possible types of signals from the mitochondrial alternative pathway may regulate carotenogenesis in $C$. zofingiensis: ROS and organic acids from the tricarboxylic acid cycle (TCA) cycle. Chemically generated ROS induce carotenogenesis and astaxanthin formation in C. zofingiensis (Ip and Chen 2005a, Li et al. 2009). Singlet oxygen specifically induced $C r t R-b$ whereas hydroxyl radical upregulated $P D S$ and $B K T$, suggesting their distinct roles in regulating carotenogenesis in C. zofingiensis (Ip and Chen 2005a). In corroboration, the fatty acid synthesis genes $S A D$ (coding for stearoyl acyl carrier protein) and $B C$ (coding for biotin carboxylase) seemed to be associated with ROS (Liu et al. 2012). On the other hand, TCA cycle acids such as citrate may act as a signal at the gene expression level to induce mitochondrial alternative pathway respiration to facilitate carotenogenesis (Vanlerberghe and McIntosh 1996). In C. zofingiensis, citrate was shown to induce $B K T$ expression and astaxanthin synthesis independent of ROS formation, suggesting that the mitochondrial alternative pathway mediates $B K T$ expression through modulation of TCA cycle with citrate as a signal molecule (Li et al. 2008a). 


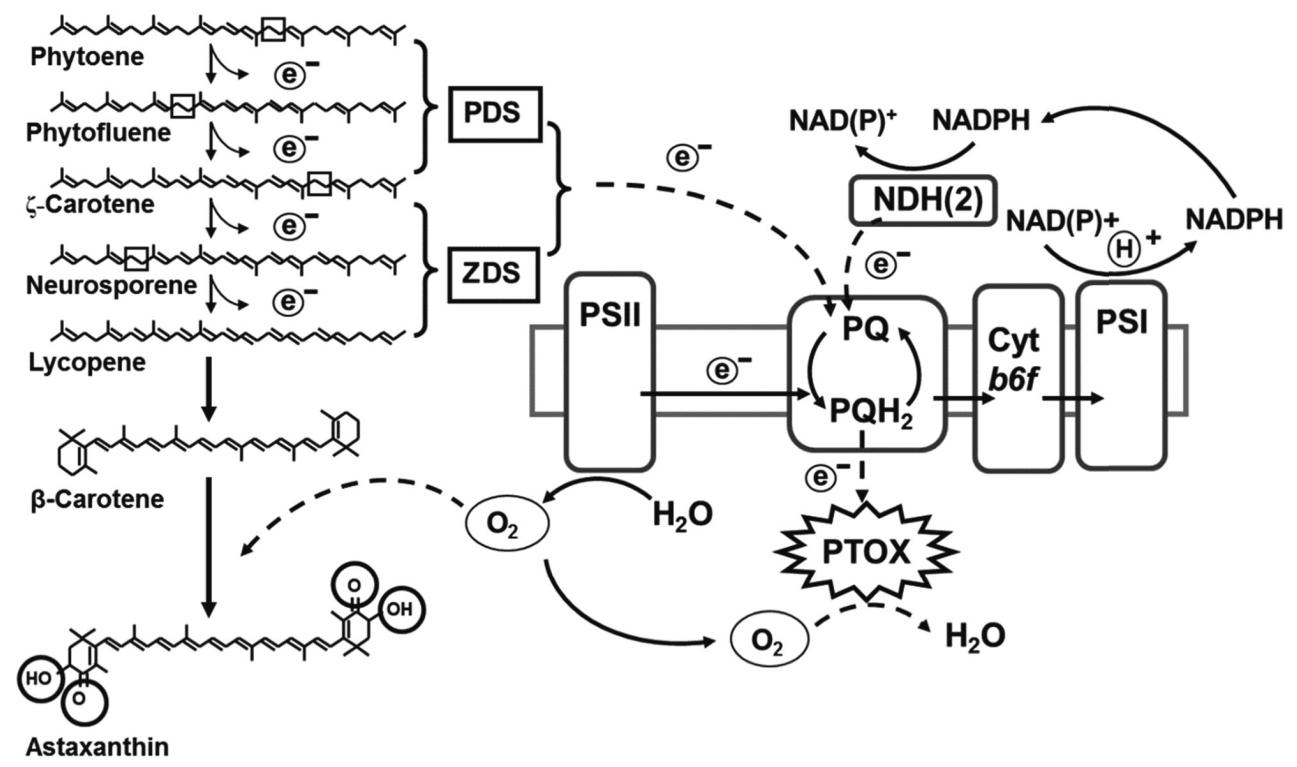

Fig. 2. Multiple roles of the astaxanthin biosynthesis in protecting microalgae against oxidative stress. Astaxanthin biosynthesis pathway functions to 1) reduce subcellular oxygen levels via synthesis of oxygen-rich astaxanthin and its esters; 2) convert photosynthetically evolved oxygen into water via a coupled electron transport from carotenoid desaturation steps (phytoene desaturase [PDS] and $\zeta$-carotene desaturase [ZDS]) to the plastoquinone pool to plastid terminal oxidase (PTOX), whereas astaxanthin itself; 3) serves as a "sunshade" to reduce excess light illumination on the photosystems; 4) functions as a powerful antioxidant against reactive oxygen species. PS, photosystem; PQ, plastoquinone. Modified and reproduced from Li (2007) with permission from the University of Hong Kong.

\section{Crosstalk between astaxanthin synthesis path- way and photosynthetic electron transfer chain}

In H. pluvialis, the nuclear carotenoid biosynthetic genes were suggested to be regulated by the redox state of the PQ pool at the photosynthetic electron transfer chain, as carotenoid biosynthesis genes are up-regulated under high PSII excitation pressure (PQ reduced) (Steinbrenner and Linden 2003), and the inhibition of photosynthesis by photosynthetic electron transport inhibitor DCMU abolished the up-regulation of the PSY gene induced by high light (Steinbrenner and Linden 2001). However, how the signal of redox state of the photosynthetic electron transport chain can pass the chloroplast envelope and regulate nuclear gene expression is not known. It was proposed that protein phosphorylation cascade may be a possible mechanism to mediate this retrograde signaling process (Chandok et al. 2001, Chen et al. 2004). Furthermore, the chlorophyll biosynthesis precursors, Mg-protoporphyrin IX and Mg-protoporphyrin-methylesters, were proposed to play a role in chloroplast-to-nucleus retrograde signaling (Nott et al. 2006).

Besides the redox state of $\mathrm{PQ}$, other photosynthetic signals such as ROS, trans-thylakoid $\mathrm{pH}$ gradient and the stoichiometry of PSI and PSII may also contribute to the photosynthetic regulation of carotenogenesis gene ex- pression (Fey et al. 2005). Recently, a transcriptomic analysis has identified over 2,000 genes regulated under high irradiance conditions, providing therefore more candidate genes for further study of the crosstalk between photosynthesis and carotenogenesis gene expression in $H$. pluvialis (Kim et al. 2011).

In addition to the regulation at the gene expression level, metabolic coupling between carotenoid and photosynthetic electron transport may be present in H. pluvialis. The electrons produced in the sequential desaturation reactions catalyzed by PDS and ZDS can be delivered to the PQ pool, which is in turn oxidized by PTOX (Fig. 2). Two genes encoding PTOX (PTOX1 and PTOX2) in H. pluvialis have been cloned and PTOX1 was found to be coregulated with the carotenoid biosynthesis genes under various environmental stress conditions (Li et al. 2008b, 2010, Wang et al. 2009). Functional analysis of PTOX of Chlamydomonas also suggested that PTOX1 was responsible for regenerating oxidized PQ for phytoene desaturation (Houille-Vernes et al. 2011).

\section{Crosstalk between astaxanthin and fatty acid biosynthesis pathways}

In H. pluvialis, over 90\% astaxanthin molecules are esterified with fatty acids (Yuan et al. 1997). Oleic acid is 
the major fatty acid species that is conjugated to astaxanthin molecules (Holtin et al. 2009). A linear correlation between the cellular fatty acid content and astaxanthin content in H. pluvialis under stress conditions has been reported in several independent studies (Zhekisheva et al. 2002, Chen 2007), leading to a hypothesis that the biosynthesis of astaxanthin and fatty acids are coupled in this organism. This hypothesis was further supported by the fact that with the addition of the fatty acid synthesis inhibitor cerulenin or the carotenoid synthesis inhibitor norflurazon, both astaxanthin and fatty acid biosynthesis were abolished (Schoefs et al. 2001, Chen 2007). Astaxanthin esterification must be the reaction that links the two pathways. Acyl-CoA, the presumable substrate for astaxanthin esterification, when in excess, may feedbackinhibit acetyl-CoA carboxylase, a rate-limiting enzyme for fatty acid synthesis (Ohlrogge and Jaworski 1997). Presumably, the inhibition of astaxanthin synthesis by norflurazon may result in building up relatively excess amounts of acyl-CoA which may in turn inhibit fatty acid synthesis through a feedback inhibition mechanism. Likewise, the enzymes responsible for astaxanthin synthesis may also be under feedback control when fatty acid biosynthesis is inhibited by cerulenin. Astaxanthin esterification, the key point for flux control, needs to be further characterized at the gene, enzyme and subcellular levels.

\section{Physiological role of astaxanthin biosynthesis}

In algae and higher plants, ROS are mainly generated through photosynthesis in chloroplasts (Asada 2006). Under stress conditions, e.g., high light, nutrient starvation, and high salinity, the imbalance between generation and detoxification of ROS within the chloroplast may cause photooxidative stress.

Astaxanthin was proposed to act as a "sunshade" to reduce the penetration of blue light into the chloroplast, thereby reducing photooxidative damage of the photosystems by excessive light (Hagen et al. 1994). Astaxanthin could also act as a physical-chemical barrier to prevent DNA, RNA, enzymes and membrane lipids from ROS attack (Hagen et al. 1993, Kobayashi et al. 1997, Kobayashi and Sakamoto 1999). Astaxanthin esters were shown to scavenge ROS, e.g., the superoxide anion radicals $\left(\mathrm{O}_{2}{ }^{-}\right)$ and singlet oxygen $\left({ }^{1} \mathrm{O}_{2}\right)$ and thus may act as an antioxidant agent against ROS (Kobayashi et al. 1997, Kobayashi and Sakamoto 1999).

The photoprotective role of astaxanthin has been challenged since astaxanthin-rich cysts of $H$. pluvialis were equally or more susceptible to high light stress than astaxanthin-free vegetative cells (Fan et al. 1998). These authors suggest that the multiple intermediate reactions in astaxanthin synthesis pathway may consume excessive electrons and thus prevent over-reduction of the photosynthetic electron transport chain and reduce the production of ROS. As such, astaxanthin was proposed as an end-product rather than the protective agent itself in the stress response process (Fan et al. 1998).

More recently, Li et al. (2008b) proposed that astaxanthin biosynthesis exerts multilevel protective roles against photooxidative stress. In addition to the protective roles summarized above, astaxanthin biosynthesis protects $H$. pluvialis cells from stress through the consumption of molecular oxygen (Li et al. 2008b). Up to $10 \%$ of molecular oxygen evolved from photosynthesis under stress is consumed by astaxanthin synthesis via two distinct routes: 1) use oxygen as a substrate for ketocarotenoids formation and 2) convert oxygen generated from photosynthesis and electrons from the carotenoid desaturation steps to water by PTOX connected to the PQ pool. Decrease in subcellular molecular oxygen concentration reduces the substrate available for oxygen dependent ROS formation, as is the case for the alternative oxidase which reduces the $\mathrm{O}_{2}$ concentration and prevents electrons from reducing $\mathrm{O}_{2}$ to $\mathrm{O}_{2}^{-1}$ (Mittler 2002). Re-oxidation of the PQ pool by PTOX may further relax the photosynthetic electron transport chain and reduce ROS formation (Li et al. $2008 b$ ). The multiple roles of the astaxanthin biosynthesis in protecting microalgae against oxidative stress are summarized in Fig. 2 (Li 2007).

In addition to the ability to accumulate large amounts of astaxanthin, microalgae such as $H$. pluvialis have evolved other mechanisms for ROS scavenging, for example, by the antioxidative enzyme defense system. Proteomics analysis revealed several superoxide dismutases (SOD), peroxidases, and catalases were up-regulated in response to photooxidative stress (Wang et al. 2004a, Tran et al. 2009b). Transcriptomic analysis revealed that the up-regulation of SOD, catalase, and glutathione peroxidase genes occurred under high light and nutrient starvation conditions (Eom et al. 2006, Kim et al. 2011, Wang et al. 2011). All the antioxidative enzymes studied showed a transient up-regulation pattern and then reverted to the basal or below basal level, suggesting that the enzyme defense system is an early response mechanism to oxidative stress, and as stress persists, the cells will adopt astaxanthin biosynthesis and accumulation as a long-term defense mechanism (Wang et al. 2004a). 


\section{BIOTECHNOLOGICAL IMPLICATIONS}

\section{Metabolic engineering for enhanced carotenoid production}

Many higher plants exhibit enzymatic activity of carotenoid hydroxylase but not $\beta$-carotene ketolase, and as a result, they produce 3-hydroxy carotenoids (e.g., lutein and zeaxanthin) but no 4-ketocarotenoids such as astaxanthin. Introduction of a Haematococcus $\beta$-carotene ketolase gene $(B K T)$ into a tobacco plant resulted in moderate accumulation of astaxanthin (ca. $840 \mu \mathrm{g} \mathrm{g}^{-1} \mathrm{dwt}$ ) in the nectary tissue (Mann et al. 2000). A similar effort was made with a carrot plant, resulting in a comparable amount (ca. $916 \mu \mathrm{g} \mathrm{g}^{-1} \mathrm{dwt}$ ) of astaxanthin in root tissues (Jayaraj et al. 2008). Expression of a Chlamydomonas BKT into Arabidopsis led to the accumulation of greater amounts of astaxanthin (up to 2,000 $\mu \mathrm{g} \mathrm{g}^{-1} \mathrm{dwt}$ ) in leaves of the transgenic plant, whereas the expression of C. zofingiensis BKT only resulted in $240 \mu \mathrm{g}$ astaxanthin $\mathrm{g}^{-1} \mathrm{dwt}$ (Zhong et al. 2011). A greater amount of astaxanthin $\left(1,600 \mu \mathrm{g} \mathrm{g}^{-1} \mathrm{dwt}\right)$ in leaves of a transgenic tobacco was also obtained when the Chlamydomonas BKT was introduced (Huang et al. 2012), suggesting that the catalytic capacity of BKT is species-specific (Zhong et al. 2011). A much greater amount of astaxanthin $\left(5,440 \mu \mathrm{g} \mathrm{g}^{-1} \mathrm{dwt}\right)$ was obtained in tobacco leaves when a $B K T$ and a $C r t R-b$ gene from a marine bacterium Brevundimonas sp. were co-transformed into the plant plastid genome (Hasunuma et al. 2008).

Lack of dominant selectable markers has hindered the genetic engineering of astaxanthin production in microalgae. Recently, a stable nuclear transformation system has been established in H. pluvialis (Steinbrenner and Sandmann 2006) and in C. zofingiensis (Huang et al. 2008). A modified version of PDS gene in H. pluvialis and C. zofingiensis has been developed as a dominant selectable marker and was transformed into these two species through biolistic transformation (Steinbrenner and Sandmann 2006, Huang et al. 2008, Liu et al. 2009). Agrobacterium-mediated transformation has also been reported toexpress foreign genes in Haematococcus (Kathiresan et al. 2009). The newly developed genetic transformation tools for $H$. pluvialis and C. zofingiensis will provide metabolic engineering opportunities for overproduction of astaxanthin in these two and perhaps other microalgae. One possible application is to enhance astaxanthin production in H. pluvialis or C. zofingiensis by overexpressing $P S Y$ and $C r t R-b$ genes, which previously have been shown to be possible rate-limiting steps for astaxanthin synthesis in H. pluvialis (Li et al. 2008b, 2010).
An alternative strategy for enhancing astaxanthin production in microalgae is through random mutagenesis. Through chemical mutagenesis, several Haematococcus mutants with improved phenotypic traits have been generated, and one such example is Haematococcus astaxanthin-hyper-accumulating mutant strain MT 2877 (Hu et al. 2008). In the early vegetative growth stage, MT 2877 was identical to the wild type with respect to cell morphology and physiology. However, the mutant grew faster under stress conditions with about $100 \%$ more viable cells. MT 2877 also accumulated $100 \%$ more astaxanthin, leading to a 4 -fold increase in volumetric astaxanthin productivity compared to the wild type (Hu et al. 2008). Earlier, two H. pluvialis cell wall deficient mutants, MT 537 and MT 2978, have been generated by chemical mutagenesis (Wang et al. 2005). Haematococcus wild type cells possess thick and rigid cell walls that reduce considerably the bioavailability of astaxanthin to human and animals if whole Haematococcus cells are consumed without cell disruption by chemical or physical means. The cell wall mutants were demonstrated to significantly improve the bioavailability of astaxanthin. Production of astaxanthin using the cell wall mutants may also reduce downstream processing costs whereby eliminating or minimizing the cell wall disruption step which is otherwise required for wild type Haematococcus (Wang et al. 2005, Hu et al. 2006). Some mating-based breeding techniques can also be applied to Haematococcus to improve existing or introduce new desirable traits, given that many Haematococcus strains vary in photosynthesis efficiency, growth, astaxanthin content, and susceptibility to oxidative stress or parasite attack. Any efforts along this line would be valuable.

\section{Effects of environmental factors on growth and astaxanthin production}

Growth and carotenogenesis of $H$. pluvialis are regulated by various environmental factors, such as light, temperature and nutrients. The maximal specific growth rate of $H$. pluvialis is $0.054 \mathrm{~h}^{-1}$, corresponding to a doubling time of 12-13 h. Such a high growth potential occurs only under favorable growth conditions, e.g., low light irradiance $\left(20-50 \mu \mathrm{E} \mathrm{m}^{-2} \mathrm{~s}^{-1}\right)$, optimal temperature $\left(25-28^{\circ} \mathrm{C}\right)$ and replete nutrients (Fan et al. 1994). On the contrary, high light, high temperature, and nutrient deprivation (e.g., nitrogen and phosphorus) induce astaxanthin synthesis while retarding cell division. High light is one of the most effective factors to stimulate astaxanthin synthesis and thus is often applied to increase astaxanthin produc- 
tion (Choi et al. 2002). High temperature is rarely implemented to induce astaxanthin production, as it was reported to severely reduce biomass yield and thus overall reduced astaxanthin productivity (Tjahjono et al. 1994).

Carotenogenesis in H. pluvialis can be stimulated by a variety of metal ions such as $\mathrm{Fe}^{2+}, \mathrm{Mn}^{2+}$, and $\mathrm{Cd}^{2+}$ (Harker et al. 1996b). All the tested metals exerted inhibitory effect on cell growth except $\mathrm{Fe}^{2+}$. $\mathrm{Fe}^{2+}$ was suggested to stimulate ROS (especially hydroxyl radicals, HO-) production via the Fenton reaction, which in turn trigger astaxanthin synthesis (Tjahjono et al. 1994). This speculation was supported by several lines of evidence: EDAT-chelated $\mathrm{FeCl}_{3} \cdot 6 \mathrm{H}_{2} \mathrm{O}$ (a form of iron which does not cause Fenton reaction) did not induce astaxanthin synthesis, whereas potassium iodide with a capability of scavenging HO- and thus abolishing $\mathrm{Fe}^{2+}$-induced astaxanthin synthesis in $H$. pluvialis (Boussiba and Vonshak 1991, Kobayashi et al. 1993).

Astaxanthin synthesis in $H$. pluvialis can also be induced by salinity stress. $\mathrm{NaCl}(0.1-0.5 \%, \mathrm{w} / \mathrm{w})$ was used to increase astaxanthin accumulation in laboratory cultures (Harker et al. 1996a, Sarada et al. 2002). However, relatively high concentrations of $\mathrm{NaCl}(0.6-0.8 \%, \mathrm{w} / \mathrm{w})$ may cause severe cell mortality, in particularly for flagellated zoospores, which limits the implementation of this strategy in large-scale Haematococcus culture (Harker et al. 1996a, Sarada et al. 2002, Cifuentes et al. 2003).

\section{Mass cultivation of Haematococcus pluvialis and Chlorella zofingiensis}

Photoautotrophic culture. Photoautotrophic culture of $H$. pluvialis is carried out in open raceway ponds and closed photobioreactors (Olaizola 2000, Carvalho et al. 2006, Ugwu et al. 2008). Because the culture conditions for maximum growth and maximum astaxanthin content are mutually exclusive, a two-stage batch culture mode is commonly adopted for mass cultivation of $H$. pluvialis. In the first stage (also called 'green stage' because the cells are green), the cells are maintained in a nutrient-replete growth medium and exposed to low light intensity to promote biomass production. When the cells enter into the stationary growth phase, the culture is then transited into the second stage (also called 'red stage' because the cells are turning red from green) where the cells are subjected to high light intensity and nutrient deprivation to induce astaxanthin production. The reported biomass productivities range from 0.03 to $0.6 \mathrm{~g} \mathrm{~L}^{-1} \mathrm{~d}^{-1}$ at the green stage and from 0.01 to $0.58 \mathrm{~g} \mathrm{~L}^{-1} \mathrm{~d}^{-1}$ at the red stage, and astaxanthin productivity ranged from 0.4 to $17 \mathrm{mg} \mathrm{L}^{-1} \mathrm{~d}^{-1}$
(Boussiba et al. 1992, Olaizola 2000, Fábregas et al. 2001, Aflalo et al. 2007).

During the first two days of the red-stage, considerable cell death (or photo-bleach) occurs, primarily to flagellated zoospores, due to their higher susceptibility to photooxidative stress than non-motile palmella cells (Harker et al. 1996a, Sarada et al. 2002, Han et al. 2012). To prevent or reduce cell mortality caused by high light and nutrient deprivation, the initial cell concentration in the culture must be optimized (Wang et al. 2013). The highest astaxanthin productivity of $17.5 \mathrm{mg} \mathrm{L}^{-1} \mathrm{~d}^{-1}$ was obtained at the red stage where an optimal initial biomass density of $0.8 \mathrm{~g} \mathrm{~L}^{-1} \mathrm{dwt}$ was applied to an outdoor glass column photobioreactor (Wang et al. 2013). Greater tolerance of palmella than zoospores to photooxidative stress resulted in more than 10-fold increase in astaxanthin productivity (Choi et al. 2011).

Besides the two-stage culture mode, a single-stage cultivation mode has also been tested to produce astaxanthin in flagellates of some $H$. pluvialis strains in a chemostat system (Del Río et al. 2005, 2008, García-Malea et al. 2009). Under optimal light irradiance, nutrient concentration and dilution rate, algal biomass productivities of $0.7-1.9 \mathrm{~g} \mathrm{~L}^{-1} \mathrm{~d}^{-1}$ were obtained, corresponding to an astaxanthin productivity of $5.6-21 \mathrm{mg} \mathrm{L}^{-1} \mathrm{~d}^{-1}$. The technical and economic feasibilities of this single-stage culture mode for mass culture of $H$. pluvialis remain to be seen.

Although a number of Chlorella species and strains have been tested for carotenoid production on laboratory scales, little has been done on a large-scale (Liu et al. 2011).

Heterotrophic and mixotrophic culture modes. H. pluvialis is capable of utilize organic carbon for growth in the absence of light, which provides the means of heterotrophic and mixotrophic cultivation for astaxanthin production. Under heterotrophic conditions, $H$. pluvialis grows at a relatively low growth rate $\left(0.22 \mathrm{~d}^{-1}\right)$ and accumulates ca. 0.5\% dwt of astaxanthin (Kobayashi et al. 1992). Growth and astaxanthin production can be enhanced under mixotrophic culture conditions. A final cell density of 0.9-2.65 g L-1 and a maximum astaxanthin content of $1-2 \%$ dwt were obtained from mixotrophic cultures of $H$. pluvialis (Chen et al. 1997, Zhang et al. 1999, Wang et al. 2004a). A heterophotric-photoautotrophic culture mode was also explored where heterotrophic culture produced algal biomass, while astaxanthin production was induced during photoautotrophic culture. As a result, a very high cellular astaxanthin content of $7 \%$ by dwt, but low astaxanthin productivities of 4.4-6.5 $\mathrm{mg} \mathrm{L}^{-1} \mathrm{~d}^{-1}$ were obtained (Hata et al. 2001, Kang et al. 2005). 
Much higher cell density can be achieved in heterotrophic culture of C. zofingiensis. For example, high cell concentrations of $10 \mathrm{~g} \mathrm{~L}^{-1}$ (Ip and Chen 2005b) and $53 \mathrm{~g}$ $\mathrm{L}^{-1}$ (Sun et al. 2008) were achieved in C. zofingiensis cultures fed with glucose with a maximum specific growth rate of $1.03 \mathrm{~d}^{-1}$. A moderate astaxanthin yield of $12.5 \mathrm{mg}$ $\mathrm{L}^{-1}$ was obtained in a mixotrophic culture of C. zofingiensis (Ip et al. 2004), and the yield was increased to $32 \mathrm{mg} \mathrm{L}^{-1}$ in a glucose fed-batch culture. However, the astaxanthin productivity is low ranging from 0.9 to $2 \mathrm{mg} \mathrm{L}^{-1} \mathrm{~d}^{-1}$ (Sun et al. 2008).

Microbial contamination and crop protection. Mass culture of Haematococcus can be contaminated by fungal parasites and zooplanktonic predators (e.g., amoebas, ciliates, and rotifers), as well as other microalgae and cyanobacteria, resulting in reduced biomass yield and quality, and sometimes loss of culture all together. A parasitic blastoclad fungus identified as Paraphysoderma sedebokerensis is the most devastating disease responsible for reduced astaxanthin productivity and frequent culture collapses (Hoffman et al. 2008, Gutman et al. 2009). Hoffman et al. (2008) made detailed observations on the infection of Haematococcus culture by the parasite P. sedebokerensis (Hoffman et al. 2008). During the infection process, healthy green culture turns dark brown, accompanied by the formation of large clumps consisting of living cells, cell debris, and particulate organic matters. A more vivid red color of culture can be first observed if infection occurred at the red stage, followed by gradual bleaching of algal cells (Hoffman et al. 2008).

The infection process can be somewhat inhibited in the presence of lectin-like carbohydrate-binding proteins, which were assumed to bind to the surface of palamelloids and aplanospores of Haematococcus (Gutman et al. 2011). The recognition between the blastoclad parasite and Haematococcus cells was suggested to be a lectin-N-acetyl-D-glucosamine interaction. More research along this line may better understand the molecular and cellular mechanisms by which P. sedebokerensis recognizes and infects Haematococcus, which may in turn lead to develop a biology-inspired method to prevent or treat the fungal disease in the mass culture of Haematococcus or other related species.

\section{Downstream processes and product formation}

Haematococcus aplanospores can be readily harvested by sedimentation and centrifugation prior to spray. If whole Haematococcus cells are a final form of product, Haematococcus pastes from centrifugation is usually pretreated by a mechanical means to disrupt the rigid cell walls, followed by a spray-drying process to produce dry biomass powder. If astaxanthin is the final product, a conventional solvent-based extraction method or supercritical fluid extraction can be applied to wet or dry Haematococcus biomass to obtain concentrated astaxanthin extracts (Bubrick 1991, Mendes-Pinto et al. 2001, Nobre et al. 2006, Sarada et al. 2006, Krichnavaruk et al. 2008).

\section{CONCLUSIONS AND PERSPECTIVES}

The occurrence and accumulation of astaxanthin in microalgae is a strategy to cope with oxidative stress. Astaxanthin synthesis pathways interact with multiple metabolic pathways such as the photosynthetic electron transport, fatty acid synthesis, and ROS generation. However, the molecular mechanisms mediating the crosstalk between astaxanthin synthesis and related metabolic pathways remain elusive. Recent progress in genome sequencing and genetic toolbox development of microalgae has made it possible to further address these questions. The new knowledge obtained from the studies of astaxanthin biosynthesis and stress response will be applied for enhanced astaxanthin production. Until now, H. pluvialis is still the best producer for astaxanthin although the biomass productivity is relatively low. Future efforts should focus on increasing biomass production of $H$. pluvialis or screening for and manipulating other fast-growing algal strains for enhanced astaxanthin production along with improved phenotypic traits for various commercial applications.

\section{ACKNOWLEDGEMENT}

The first two authors contributed equally to this work.

\section{REFERENCES}

Aflalo, C., Meshulam, Y., Zarka, A. \& Boussiba, S. 2007. On the relative efficiency of two-vs. one-stage production of astaxanthin by the green alga Haematococcus pluvialis. Biotechnol. Bioeng. 98:300-305.

Asada, K. 2006. Production and scavenging of reactive oxygen species in chloroplasts and their functions. Plant Physiol. 141:391-396.

Bar, E., Rise, M., Vishkautsan, M. \& Arad, S. 1995. Pigment and structural changes in Chlorella zofingiensis upon 
light and nitrogen stress. J. Plant Physiol. 146:527-534.

Barbosa, M. J., Morais, R. \& Choubert, G. 1999. Effect of carotenoid source and dietary lipid content on blood astaxanthin concentration in rainbow trout (Oncorhynchus mykiss). Aquaculture 176:331-341.

Bidigare, R. R., Ondrusek, M. E., Kennicutt, M. C., Iturriaga, R., Harvey, H. R., Hoham, R. W. \& Macko, S. A. 1993. Evidence for a photoprotective function for secondary carotenoids of snow algae. J. Phycol. 29:427-434.

Boussiba, S. 2000. Carotenogenesis in the green alga Haematococcus pluvialis: cellular physiology and stress response. Physiol Plant. 108:111-117.

Boussiba, S., Bing, W., Yuan, J. -P., Zarka, A. \& Chen, F. 1999. Changes in pigments profile in the green alga Haeamtococcus pluvialis exposed to environmental stresses. Biotechnol. Lett. 21:601-604.

Boussiba, S., Fan, L. \& Vonshak, A. 1992. Enhancement and determination of astaxanthin accumulation in green alga Haematococcus pluvialis. Methods Enzymol. 213:386-391.

Boussiba, S. \& Vonshak, A. 1991. Astaxanthin accumulation in the green alga Haematococcus pluvialis. Plant Cell Physiol. 32:1077-1082.

Brinda, B. R., Sarada, R., Kamath, B. S., \& Ravishankar, G. A. 2004. Accumulation of astaxanthin in flagellated cells of Haematococcus pluvialis - cultural and regulatory aspects. Curr. Sci. (Bangalore) 87:1290-1294.

Bubrick, P. 1991. Production of astaxanthin from Haematococcus. Bioresour. Technol. 38:237-239.

Carol, P., Stevenson, D., Bisanz, C., Breitenbach, J., Sandmann, G., Mache, R., Coupland, G. \& Kuntz, M. 1999. Mutations in the Arabidopsis gene immutans cause a variegated phenotype by inactivating a chloroplast terminal oxidase associated with phytoene desaturation. Plant Cell 11:57-68.

Carvalho, A. P., Meireles, L. A. \& Malcata, F. X. 2006. Microalgal reactors: a review of enclosed system designs and performances. Biotechnol. Prog. 22:1490-1506.

Chandok, M. R., Sopory, S. K. \& Oelmüller, R. 2001. Cytoplasmic kinase and phosphatase activities can induce PsaF gene expression in the absence of functional plastids: evidence that phosphorylation/dephosphorylation events are involved in interorganellar crosstalk. Mol. Gen. Genet. 264:819-826.

Chen, F., Chen, H. \& Gong, X. 1997. Mixotrophic and heterotrophic growth of Haematococcus lacustris and rheological behaviour of the cell suspensions. Bioresour. Technol. 62:19-24.

Chen, G. 2007. Lipid and fatty acid composition and their biosyntheses in relation to carotenoid accumulation in the microalgae Nitzschia laevis (Bacillariophyceae) and Haematococcus pluvialis (Chlorophyceae). Ph.D. dissertation, The University of Hong Kong, Hong Kong, 150 pp.

Chen, Y. -B., Durnford, D. G., Koblizek, M. \& Falkowski, P. G. 2004. Plastid regulation of Lhcbl transcription in the chlorophyte alga Dunaliella tertiolecta. Plant Physiol. 136:3737-3750.

Choi, Y. E., Yun, Y. -S. \& Park, J. M. 2002. Evaluation of factors promoting astaxanthin production by a unicellular green alga, Haematococcus pluvialis, with fractional factorial design. Biotechnol. Prog. 18:1170-1175.

Choi, Y. -E., Yun, Y. -S., Park, J. M. \& Yang, J. -W. 2011. Determination of the time transferring cells for astaxanthin production considering two-stage process of Haematococcus pluvialis cultivation. Bioresour. Technol. 102:11249-11253.

Cifuentes, A. S., González, M. A., Vargas, S., Hoeneisen, M. \& González, N. 2003. Optimization of biomass, total carotenoids and astaxanthin production in Haematococcus pluvialis Flotow strain Steptoe (Nevada, USA) under laboratory conditions. Biol. Res. 36:343-357.

Cordero, B. F., Couso, I., León, R., Rodríguez, H. \& Vargas, M. Á. 2011. Enhancement of carotenoids biosynthesis in Chlamydomonas reinhardtii by nuclear transformation using a phytoene synthase gene isolated from Chlorella zofingiensis. Appl. Microbiol. Biotechnol. 91:341-351.

Cordero, B. F., Couso, I., León, R., Rodriguez, H. \& Vargas, M. A. 2012. Isolation and characterization of a lycopene epsilon-cyclase gene of Chlorella (Chromochloris) zofingiensis: regulation of the carotenogenic pathway by nitrogen and light. Mar. Drugs 10:2069-2088.

Cordero, B. F., Obraztsova, I., Martín, L., Couso, I., León, R., Vargas, M. A. \& Rodriguez, H. 2010. Isolation and characterization of a lycopene $\beta$-cyclase gene from the astaxanthin-producing green alga Chlorella zofingiensis (Chlorophyta). J. Phycol. 46:1229-1238.

Cunningham, F. X. \& Gantt, E. 1998. Genes and enzymes of carotenoid biosynthesis in plants. Annu. Rev. Plant Physiol. Plant Mol. Biol. 49:557-583.

Cunningham, F. X., Pogson, B., Sun, Z., McDonald, K. A., DellaPenna, D. \& Gantt, E. 1996. Functional analysis of the beta and epsilon lycopene cyclase enzymes of Arabidopsis reveals a mechanism for control of cyclic carotenoid formation. Plant Cell 8:1613-1626.

Damiani, M. C., Leonardi, P. I., Pieroni, O. I. \& Cáceres, E. J. 2006. Ultrastructure of the cyst wall of Haematococcus pluvialis (Chlorophyceae): wall development and behaviour during cyst germination. Phycologia 45:616623.

Del Campo, J. A., Rodríguez, H., Moreno, J., Vargas, M. Á., Ri- 
vas, J. \& Guerrero, M. G. 2004. Accumulation of astaxanthin and lutein in Chlorella zofingiensis (Chlorophyta). Appl. Microbiol. Biotechnol. 64:848-854.

Del Río, E., Acién, F. G., García-Malea, M. C., Rivas, J., MolinaGrima, E. \& Guerrero, M. G. 2005. Efficient one-step production of astaxanthin by the microalga Haematococcus pluvialis in continuous culture. Biotechnol. Bioeng. 91:808-815.

Del Río, E., Acién, F. G., García-Malea, M. C., Rivas, J., Molina-Grima, E. \& Guerrero, M. G. 2008. Efficiency assessment of the one-step production of astaxanthin by the microalga Haematococcus pluvialis. Biotechnol. Bioeng. 100:397-402.

Disch, A., Schwender, J., Müller, C., Lichtenthaler, H. K. \& Rohmer, M. 1998. Distribution of the mevalonate and glyceraldehyde phosphate/pyruvate pathways for isoprenoid biosynthesis in unicellular algae and the cyanobacterium Synechocystis PCC 6714. Biochem. J. 333:381388.

Droop, M. R. 1954. Conditions governing haematochrome formation and loss in the alga Haematococcus pluvialis Flotow. Arch. Microbiol. 20:391-397.

Elliot, A. M. 1934. Morphology and life history of Haematococcus pluvialis. Arch. Protistenk 82:250-272.

Eom, H., Lee, C. G. \& Jin, E. 2006. Gene expression profile analysis in astaxanthin-induced Haematococcus pluvialis using a cDNA microarray. Planta 223:1231-1242.

Fábregas, J., Otero, A., Maseda, A. \& Domínguez, A. 2001. Two-stage cultures for the production of astaxanthin from Haematococcus pluvialis. J. Biotechnol. 89:65-71.

Fan, L., Vonshak, A. \& Boussiba, S. 1994. Effect of temperature and irradiance on growth of Haematococcus pluvialis (Chlorophyceae). J. Phycol. 30:829-833.

Fan, L., Vonshak, A., Zarka, A. \& Boussiba, S. 1998. Does astaxanthin protect Haematococcus against light damage? Z. Naturforsch. C 53:93-100.

Fey, V., Wagner, R., Bräutigam, K. \& Pfannschmidt, T. 2005. Photosynthetic redox control of nuclear gene expression. J. Exp. Bot. 56:1491-1498.

Fraser, P. D., Shimada, H. \& Misawa, N. 1998. Enzymic confirmation of reactions involved in routes to astaxanthin formation, elucidated using a direct substrate in vitro assay. Eur. J. Biochem. 252:229-236.

García-Malea, M. C., Acién, F. G., Del Río, E., Fernández, J. M., Cerón, M. C., Guerrero, M. G. \& Molina-Grima, E. 2009. Production of astaxanthin by Haematococcus pluvialis: taking the one-step system outdoors. Biotechnol. Bioeng. 102:651-657.

Green, J. 1963. Occurrence of astaxanthin in euglenoid Trachelomonas volvocina. Comp. Biochem. Physiol. 9:313-
316.

Grunewald, K., Eckert, M., Hirschberg, J. \& Hagen, C. 2000. Phytoene desaturase is localized exclusively in the chloroplast and up-regulated at the mRNA level during accumulation of secondary carotenoids in Haematococcus pluvialis (Volvocales, Chlorophyceae). Plant Physiol. 122:1261-1268.

Grünewald, K., Hirschberg, J. \& Hagen, C. 2001. Ketocarotenoid biosynthesis outside of plastids in the unicellular green alga Haematococcus pluvialis. J. Biol. Chem. 276:6023-6029.

Grung, M., Metzger, P. \& Liaaen-Jensen, S. 1994. Algal carotenoids 53: secondary carotenoids of algae 4 . Secondary carotenoids in the green alga Botryococcus braunii, race L, new strain. Biochem. Syst. Ecol. 22:25-29.

Guerin, M., Huntley, M. E. \& Olaizola, M. 2003. Haematococcus astaxanthin: applications for human health and nutrition. Trends Biotechnol. 21:210-216.

Gutman, J., Zarka, A. \& Boussiba, S. 2009. The host-range of Paraphysoderma sedebokerensis, a chytrid that infects Haematococcus pluvialis. Eur. J. Phycol. 44:509-514.

Gutman, J., Zarka, A. \& Boussiba, S. 2011. Evidence for the involvement of surface carbohydrates in the recognition of Haematococcus pluvialis by the parasitic blastoclad Paraphysoderma sedebokerensis. Fungal Biol. 115:803811.

Hagen, C., Braune, W. \& Björn, L. O. 1994. Functional aspects of secondary carotenoids in Haematococcus lacustris (Volvocales). III. Action as a sunshade. J. Phycol. 30:241248.

Hagen, C., Braune, W. \& Greulich, F. 1993. Functional-aspects of secondary carotenoids in Haematococcus lacustris [Girod] Rostafinski (Volvocales). IV. Protection from photodynamic damage. J. Photochem. Photobiol. B 20:153-160.

Hagen, C., Grünewald, K., Schmidt, S., \& Müller, J. 2000. Accumulation of secondary carotenoids in flagellates of Haematococcus pluvialis (Chlorophyta) is accompanied by an increase in per unit chlorophyll productivity of photosynthesis. Eur. J. Phycol. 35:75-82.

Hagen, C., Siegmund, S. \& Braune, W. 2008. Ultrastructural and chemical changes in the cell wall of Haematococcus pluvialis (Vovocales, Chlorophyta) during aplanospore formation. Eur. J. Phycol. 37:217-226.

Han, D., Wang, J., Sommerfeld, M. \& Hu, Q. 2012. Susceptibility and potective mechanisms of motile and nonmotile cells of Haematococcus pluvialis (Chlorophyceae) to photooxidative stress. J. Phycol. 48:693-705.

Harker, M., Tsavalos, A. J. \& Young, A. J. 1996a. Autotrophic growth and carotenoid production of Haematococcus 
pluvialis in a 30 liter air-lift photobioreactor. J. Ferment. Bioeng. 82:113-118.

Harker, M., Tsavalos, A. J. \& Young, A. J. 1996b. Factors responsible for astaxanthin formation in the chlorophyte Haematococcus pluvialis. Bioresour. Technol. 55:207214.

Hasunuma, T., Miyazawa, S. I., Yoshimura, S., Shinzaki, Y., Tomizawa, K. I., Shindo, K., Choi, S. K., Misawa, N. \& Miyake, C. 2008. Biosynthesis of astaxanthin in tobacco leaves by transplastomic engineering. Plant J. 55:857868.

Hata, N., Ogbonna, J. C., Hasegawa, Y., Taroda, H. \& Tanaka, H. 2001. Production of astaxanthin by Haematococcus pluvialis in a sequential heterotrophic-photoautotrophic culture. J. Appl. Phycol. 13:395-402.

Hershkovits, G., Dubinsky, Z. \& Katcoff, D. J. 1997. A novel homologue of the prokaryotic htrA gene is differentially expressed in the alga Haematococcus pluvialis following stress. Mol. Gen. Genet. 254:345-350.

Hoffman, Y., Aflalo, C., Zarka, A., Gutman, J., James, T. Y. \& Boussiba, S. 2008. Isolation and characterization of a novel chytrid species (phylum Blastocladiomycota), parasitic on the green alga Haermatococcus. Mycol. Res. 112:70-81.

Holtin, K., Kuehnle, M., Rehbein, J., Schuler, P., Nicholson, G. \& Albert, K. 2009. Determination of astaxanthin and astaxanthin esters in the microalgae Haematococcus pluvialis by LC-(APCI)MS and characterization of predominant carotenoid isomers by NMR spectroscopy. Anal. Bioanal. Chem. 395:1613-1622.

Houille-Vernes, L., Rappaport, F., Wollman, F. A., Alric, J. \& Johnson, X. 2011. Plastid terminal oxidase 2 (PTOX2) is the major oxidase involved in chlororespiration in Chlamydomonas. Proc. Natl. Acad. Sci. U. S. A. 108:2082020825.

Hu, Q., Sommerfeld, M. \& Lu, F. 2006. Extractability and bioavailability of the natural antioxidant astaxanthin from a green alga, Haematococcus pluvialis. WIPO Patent No. 2006107736.

Hu, Z., Li, Y., Sommerfeld, M. \& Hu, Q. 2008. Enhanced protection against oxidative stress in an astaxanthin-overproduction Haematococcus mutant (Chlorophyceae). Eur. J. Phycol. 43:365-376.

Huang, J. C., Chen, F. \& Sandmann, G. 2006a. Stress-related differential expression of multiple $\beta$-carotene ketolase genes in the unicellular green alga Haematococcus pluvialis. J. Biotechnol. 122:176-185.

Huang, J., Liu, J., Li, Y. \& Chen, F. 2008. Isolation and characterization of the phytoene desaturase gene as a potential selective marker for genetic engineering of the astaxanthin-producing green alga Chlorella zofingiensis (Chlorophyta). J. Phycol. 44:684-690.

Huang, J. C., Wang, Y., Sandmann, G. \& Chen, F. 2006b. Isolation and characterization of a carotenoid oxygenase gene from Chlorella zofingiensis (Chlorophyta). Appl. Microbiol. Biotechnol. 71:473-479.

Huang, J. C., Zhong, Y. J., Sandmann, G., Liu, J. \& Chen, F. 2012. Cloning and selection of carotenoid ketolase genes for the engineering of high-yield astaxanthin in plants. Planta 236:691-699.

Huss, V. A. R., Frank, C., Hartmann, E. C., Hirmer, M., Kloboucek, A., Seidel, B. M., Wenzeler, P. \& Kessler, E. 1999. Biochemical taxonomy and molecular phylogeny of the genus Chlorella sensu lato (Chlorophyta). J. Phycol. 35:587-598.

Ip, P. -F. \& Chen, F. 2005a. Employment of reactive oxygen species to enhance astaxanthin formation in Chlorella zofingiensis in heterotrophic culture. Process Biochem. 40:3491-3496.

Ip, P. -F. \& Chen, F. 2005b. Production of astaxanthin by the green microalga Chlorella zofingiensis in the dark. Process Biochem. 40:733-738.

Ip, P. -F., Wong, K. -H. \& Chen, F. 2004. Enhanced production of astaxanthin by the green microalga Chlorella zofingiensis in mixotrophic culture. Process Biochem. 39:17611766.

Jayaraj, J., Devlin, R. \& Punja, Z. 2008. Metabolic engineering of novel ketocarotenoid production in carrot plants. Transgenic Res. 17:489-501.

Johnson, E. A. \& An, G. -H. 1991. Astaxanthin from microbial sources. Crit. Rev. Biotechnol. 11:297-326.

Johnson, E. A. \& Schroeder, W. A. 1996. Microbial carotenoids. Adv. Biochem. Eng. Biotechnol. 53:119-178.

Kajiwara, S., Fraser, P. D., Kondo, K. \& Misawa, N. 1997. Expression of an exogenous isopentenyl diphosphate isomerase gene enhances isoprenoid biosynthesis in Escherichia coli. Biochem. J. 324:421-426.

Kajiwara, S., Kakizono, T., Saito, T., Kondo, K., Ohtani, T., Nishio, N., Nagai, S. \& Misawa, N. 1995. Isolation and functional identification of a novel cDNA for astaxanthin biosynthesis from Haematococcus pluvialis, and astaxanthin synthesis in Escherichia coli. Plant Mol. Biol. 29:343-352.

Kang, C. D., Lee, J. S., Park, T. H. \& Sim, S. J. 2005. Comparison of heterotrophic and photoautotrophic induction on astaxanthin production by Haematococcus pluvialis. Appl. Microbiol. Biotechnol. 68:237-241.

Kathiresan, S., Chandrashekar, A., Ravishankar, G. A. \& Sarada, R. 2009. Agrobacterium-mediated transformation in the green alga Haematococcus pluvialis (Chlorophyce- 
ae, Volvocales). J. Phycol. 45:642-649.

Kim, D. -K., Hong, S. -J., Bae, J. -H., Yim, N., Jin, E. S. \& Lee, C. -G. 2011. Transcriptomic analysis of Haematococcus lacustris during astaxanthin accumulation under high irradiance and nutrient starvation. Biotechnol. Bioprocess Eng. 16:698-705.

Kobayashi, M., Kakizono, T. \& Nagai, S. 1993. Enhanced carotenoid biosynthesis by oxidative stress in acetate-induced cyst cells of a green unicellular alga, Haematococcus pluvialis. Appl. Environ. Microb. 59:867-873.

Kobayashi, M., Kakizono, T., Nishio, N., Nagai, S., Kurimura, Y. \& Tsuji, Y. 1997. Antioxidant role of astaxanthin in the green alga Haematococcus pluvialis. Appl. Microbiol. Biotechnol. 48:351-356.

Kobayashi, M., Kakizono, T., Yamaguchi, K., Nishio, N. \& Nagai, S. 1992. Growth and astaxanthin formation of Haematococcus pluvialis in heterotrophic and mixotrophic conditions. J. Ferment. Bioeng. 74:17-20.

Kobayashi, M. \& Sakamoto, Y. 1999. Singlet oxygen quenching ability of astaxanthin esters from the green alga Haematococcus pluvialis. Biotechnol. Lett. 21:265-269.

Krichnavaruk, S., Shotipruk, A., Goto, M. \& Pavasant, P. 2008. Supercritical carbon dioxide extraction of astaxanthin from Haematococcus pluvialis with vegetable oils as cosolvent. Bioresour. Technol. 99:5556-5560.

Lang, N. J. 1968. Electron microscopic studies of extraplastidic astaxanthin in Haematococcus. J. Phycol. 4:12-19.

Lee, Y. -K. \& Ding, S. -Y. 1994. Cell cycle and accumulation of astaxanthin in Haematococcus lacustris (Chlorophyta). J. Phycol. 30:445-449.

Lemoine, Y. \& Schoefs, B. 2010. Secondary ketocarotenoid astaxanthin biosynthesis in algae: a multifunctional response to stress. Photosynth. Res. 106:155-177.

Leya, T., Rahn, A., Lütz, C. \& Remias, D. 2009. Response of arctic snow and permafrost algae to high light and nitrogen stress by changes in pigment composition and applied aspects for biotechnology. FEMS Microbiol. Ecol. 67:432-443.

Li, J., Zhu, D., Niu, J., Shen, S. \& Wang, G. 2011. An economic assessment of astaxanthin production by large scale cultivation of Haematococcus pluvialis. Biotechnol. Adv. 29:568-574.

Li, Y. 2007. The role of carotenogenesis in the response of the green alga Haematococcus pluvialis to oxidative stress. Ph.D. dissertation, The University of Hong Kong, Hong Kong, 157 pp.

Li, Y., Huang, J., Sandmann, G. \& Chen, F. 2008a. Glucose sensing and the mitochondrial alternative pathway are involved in the regulation of astaxanthin biosynthesis in the dark-grown Chlorella zofingiensis (Chlorophyceae).
Planta 228:735-743.

Li, Y., Huang, J., Sandmann, G. \& Chen, F. 2009. High-light and sodium chloride stress differentially regulate the biosynthesis of astaxanthin in Chlorella zofingiensis (Chlorophyceae). J. Phycol. 45:635-641.

Li, Y., Sommerfeld, M., Chen, F. \& Hu, Q. 2008b. Consumption of oxygen by astaxanthin biosynthesis: a protective mechanism against oxidative stress in Haematococcus pluvialis (Chlorophyceae). J. Plant Physiol. 165:17831797.

Li, Y., Sommerfeld, M., Chen, F. \& Hu, Q. 2010. Effect of photon flux densities on regulation of carotenogenesis and cell viability of Haematococcus pluvialis (Chlorophyceae). J. Appl. Phycol. 22:253-263.

Lichtenthaler, H. K. 1999. The 1-deoxy-D-xylulose-5-phosphate pathway of isoprenoid biosynthesis in plants. Annu. Rev. Plant Physiol. Plant Mol. Biol. 50:47-65.

Lichtenthaler, H. K., Rohmer, M. \& Schwender, J. 1997. Two independent biochemical pathways for isopentenyl diphosphate and isoprenoid biosynthesis in higher plants. Physiol. Plant. 101:643-652.

Linden, H. 1999. Carotenoid hydroxylase from Haematococcus pluvialis: cDNA sequence, regulation and functional complementation. Biochim. Biophys. Acta 1446:203212.

Liu, B. -H. \& Lee, Y. -K. 2000. Secondary carotenoids formation by the green alga Chlorococcum sp. J. Appl. Phycol. 12:301-307.

Liu, J., Huang, J. \& Chen, F. 2009. Metabolic engineering of Chlorella zofingiensis (Chlorophyta) for enhanced biosynthesis of astaxanthin. FEBS J. 276:S283.

Liu, J., Huang, J., Sun, Z., Zhong, Y., Jiang, Y. \& Chen, F. 2011. Differential lipid and fatty acid profiles of photoautotrophic and heterotrophic Chlorella zofingiensis: assessment of algal oils for biodiesel production. Bioresour. Technol. 102:106-110.

Liu, J., Sun, Z., Zhong, Y. J., Huang, J., Hu, Q. \& Chen, F. 2012. Stearoyl-acyl carrier protein desaturase gene from the oleaginous microalga Chlorella zofingiensis: cloning, characterization and transcriptional analysis. Planta 236:1665-1676.

Lorenz, R. T. \& Cysewski, G. R. 2000. Commercial potential for Haematococcus microalgae as a natural source of astaxanthin. Trends Biotechnol. 18:160-167.

Lotan, T. \& Hirschberg, J. 1995. Cloning and expression in Escherichia coli of the gene encoding $\beta$-C-4-oxygenase, that converts $\beta$-carotene to the ketocarotenoid canthaxanthin in Haematococcus pluvialis. FEBS Lett. 364:125128.

Ma, R. Y. -N. \& Chen, F. 2001. Enhanced production of free 
trans-astaxanthin by oxidative stress in the cultures of the green microalga Chlorococcum sp. Process Biochem. 36:1175-1179.

Mann, V., Harker, M., Pecker, I. \& Hirschberg, J. 2000. Metabolic engineering of astaxanthin production in tobacco flowers. Nat. Biotechnol. 18:888-892.

Mendes-Pinto, M. M., Raposo, M. F. J., Bowen, J., Young, A. J. \& Morais, R. 2001. Evaluation of different cell disruption processes on encysted cells of Haematococcus pluvialis: effects on astaxanthin recovery and implications for bio-availability. J. Appl. Phycol. 13:19-24.

Miki, W. 1991. Biological functions and activities of animal carotenoids. Pure Appl. Chem. 63:141-146.

Misawa, N. 2009. Pathway engineering of plants toward astaxanthin production. Plant Biotechnol. 26:93-99.

Mittler, R. 2002. Oxidative stress, antioxidants and stress tolerance. Trends Plant Sci. 7:405-410.

Nobre, B., Marcelo, F., Passos, R., Beirão, L., Palavra, A., Gouveia, L. \& Mendes, R. 2006. Supercritical carbon dioxide extraction of astaxanthin and other carotenoids from the microalga Haematococcus pluvialis. Eur. Food Res. Technol. 223:787-790.

Nott, A., Jung, H. S., Koussevitzky, S. \& Chory, J. 2006. Plastidto-nucleus retrograde signaling. Annu. Rev. Plant Biol. 57:739-759

Ohlrogge, J. B. \& Jaworski, J. G. 1997. Regulation of fatty acid synthesis. Annu. Rev. Plant Physiol. Plant Mol. Biol. 48:109-136.

Olaizola, M. 2000. Commercial production of astaxanthin from Haematococcus pluvialis using 25,000-liter outdoor photobioreactors. J. Appl. Phycol. 12:499-506.

Orosa, M., Torres, E., Fidalgo, P. \& Abalde, J. 2000. Production and analysis of secondary carotenoids in green algae. J. Appl. Phycol. 12:553-556.

Qin, S., Liu, G. -X. \& Hu, Z. -Y. 2008. The accumulation and metabolism of astaxanthin in Scenedesmus obliquus (Chlorophyceae). Process Biochem. 43:795-802.

Qiu, B. S. \& Li, Y. 2006. Photosynthetic acclimation and photoprotective mechanism of Haematococcus pluvialis (Chlorophyceae) during the accumulation of secondary carotenoids at elevated irradiation. Phycologia 45:117126.

Remias, D., Karsten, U., Lütz, C. \& Leya, T. 2010. Physiological and morphological processes in the alpine snow alga Chloromonas nivalis (Chlorophyceae) during cyst formation. Protoplasma 243:73-86.

Remias, D., Lütz-Meindl, U. \& Lütz, C. 2005. Photosynthesis, pigments and ultrastructure of the alpine snow alga Chlamydomonas nivalis. Eur. J. Phycol. 40:259-268.

Rise, M., Cohen, E., Vishkautsan, M., Cojocaru, M., Gottlieb,
H. E. \& Arad, S. M. 1994. Accumulation of secondary carotenoids in Chlorella zofingiensis. J. Plant Physiol. 144:287-292.

Santos, M. F. \& Mesquita, J. F. 1984. Ultrastructure study of Haematococcus lacustris (Girod.) Rostafinski (Volvocales) I. Some aspects of carotenogenesis. Cytologia 49:215-228.

Sarada, R., Bhattacharya, S., Bhattacharya, S. \& Ravishankar, G. A. 2002. A response surface approach for the production of natural pigment astaxanthin from green alga, Haematococcus pluvialis: effect of sodium acetate, culture age, and sodium chloride. Food Biotechnol. 16:107120.

Sarada, R., Vidhyavathi, R., Usha, D. \& Ravishankar, G. A. 2006. An efficient method for extraction of astaxanthin from green alga Haematococcus pluvialis. J. Agric. Food Chem. 54:7585-7588.

Schoefs, B., Rmiki, N., Rachadi, J. \& Lemoine, Y. 2001. Astaxanthin accumulation in Haematococcus requires a cytochrome P450 hydroxylase and an active synthesis of fatty acids. FEBS Lett. 500:125-128.

Steinbrenner, J. \& Linden, H. 2001. Regulation of two carotenoid biosynthesis genes coding for phytoene synthase and carotenoid hydroxylase during stress-induced astaxanthin formation in the green alga Haematococcus pluvialis. Plant Physiol. 125:810-817.

Steinbrenner, J. \& Linden, H. 2003. Light induction of carotenoid biosynthesis genes in the green alga Haematococcus pluvialis: regulation by photosynthetic redox control. Plant Mol. Biol. 52:343-356.

Steinbrenner, J. \& Sandmann, G. 2006. Transformation of the green alga Haematococcus pluvialis with a phytoene desaturase for accelerated astaxanthin biosynthesis. Appl. Environ. Microbiol. 72:7477-7484.

Sun, N., Wang, Y., Li, Y. -T., Huang, J. -C. \& Chen, F. 2008. Sugar-based growth, astaxanthin accumulation and carotenogenic transcription of heterotrophic Chlorella zofingiensis (Chlorophyta). Process Biochem. 43:12881292.

Sun, Z., Cunningham, F. X. \& Gantt, E. 1998. Differential expression of two isopentenyl pyrophosphate isomerases and enhanced carotenoid accumulation in a unicellular chlorophyte. Proc. Natl. Acad. Sci. U. S. A. 95:1148211488.

Takeda, H. 1991. Sugar composition of the cell-wall and the taxonomy of Chlorella (Chlorophyceae). J. Phycol. 27:224-232.

Tan, S., Cunningham, F. X., Youmans, M., Grabowski, B., Sun, Z. \& Gantt, E. 1995. Cytochrome f loss in astaxanthinaccumulating red cells of Haematococcus pluvialis 
(Chlorophyceae): comparison of photosynthetic activity, photosynthetic enzymes, and thylakoid membrane polypeptides in red and green cells. J. Phycol. 31:897905.

Tjahjono, A. E., Hayama, Y., Kakizono, T., Terada, Y., Nishio, N. \& Nagai, S. 1994. Hyper-accumulation of astaxanthin in a green alga Haematococcus pluvialis at elevated temperatures. Biotechnol. Lett. 16:133-138.

Tran, D., Haven, J., Qiu, W. -G. \& Polle, J. E. W. 2009a. An update on carotenoid biosynthesis in algae: phylogenetic evidence for the existence of two classes of phytoene synthase. Planta 229:723-729.

Tran, N. -P., Park, J. -K. \& Lee, C. -G. 2009b. Proteomics analysis of proteins in green alga Haematococcus lacustris (Chlorophyceae) expressed under combined stress of nitrogen starvation and high irradiance. Enzyme Microb. Technol. 45:241-246.

Triki, A., Maillard, P. \& Gudin, C. 1997. Gametogenesis in Haematococcus pluvialis Flotow (Volvocales, Chlorophyta). Phycologia 36:190-194.

Ugwu, C. U., Aoyagi, H. \& Uchiyama, H. 2008. Photobioreactors for mass cultivation of algae. Bioresour. Technol. 99:4021-4028.

Vanlerberghe, G. C. \& McIntosh, L. 1996. Signals regulating the expression of the nuclear gene encoding alternative oxidase of plant mitochondria. Plant Physiol. 111:589595.

Vechtel, B., Kahmann, U. \& Ruppel, H. G. 1992. Secondary carotenoids of Eremosphaera viridis De Bary (Chlorophyceae) under nitrogen deficiency. Bot. Acta 105:219222.

Vershinin, A. 1999. Biological functions of carotenoids: diversity and evolution. Biofactors 10:99-104.

Vidhyavathi, R., Venkatachalam, L., Sarada, R. \& Ravishankar, G. A. 2008. Regulation of carotenoid biosynthetic genes expression and carotenoid accumulation in the green alga Haematococcus pluvialis under nutrient stress conditions. J. Exp. Bot. 59:1409-1418.

Wang, B., Zarka, A., Trebst, A. \& Boussiba, S. 2003. Astaxanthin accumulation in Haematococcus pluvialis (Chlorophyceae) as an active photoprotective process under high irradiance. J. Phycol. 39:1116-1124.

Wang, C. W., Oh, M. K. \& Liao, J. C. 1999. Engineered isoprenoid pathway enhances astaxanthin production in Escherichia coli. Biotechnol. Bioeng. 62:235-241.

Wang, J., Han, D., Sommerfeld, M. R., Lu, C. \& Hu, Q. 2013. Effect of initial biomass density on growth and astaxanthin production of Haematococcus pluvialis in an outdoor photobioreactor. J. Appl. Phycol. 25:253-260.

Wang, J., Sommerfeld, M. \& Hu, Q. 2009. Occurrence and environmental stress responses of two plastid terminal oxidases in Haematococcus pluvialis (Chlorophyceae). Planta 230:191-203.

Wang, J., Sommerfeld, M. \& Hu, Q. 2011. Cloning and expression of isoenzymes of superoxide dismutase in Haematococcus pluvialis (Chlorophyceae) under oxidative stress. J. Appl. Phycol. 23:995-1003.

Wang, S. B., Chen, F., Sommerfeld, M. \& Hu, Q. 2004a. Proteomic analysis of molecular response to oxidative stress by the green alga Haematococcus pluvialis (Chlorophyceae). Planta 220:17-29.

Wang, S. B., Chen, F., Sommerfeld, M. \& Hu, Q. 2005. Isolation and proteomic analysis of cell wall-deficient Haematococcus pluvialis mutants. Proteomics 5:4839-4851.

Wang, S. B., Hu, Q., Sommerfeld, M. \& Chen, F. 2004b. Cell wall proteomics of the green alga Haematococcus pluvialis (Chlorophyceae). Proteomics 4:692-708.

Wang, Y. \& Chen, T. 2008. The biosynthetic pathway of carotenoids in the astaxanthin-producing green alga Chlorella zofingiensis. World J. Microbiol. Biotechnol. 24:29272932.

Wu, D. Y., Wright, D. A., Wetzel, C., Voytas, D. F. \& Rodermel, S. 1999. The immutans variegation locus of Arabidopsis defines a mitochondrial alternative oxidase homolog that functions during early chloroplast biogenesis. Plant Cell 11:43-55.

Yuan, J. -P., Gong, X. -D. \& Chen, F. 1997. Separation and analysis of carotenoids and chlorophylls in Haematococcus lacustris by high-performance liquid chromatography photodiode array detection. J. Agric. Food Chem. 45:1952-1956.

Zhang, X. W., Gong, X. -D. \& Chen, F. 1999. Kinetic models for astaxanthin production by high cell density mixotrophic culture of the microalga Haematococcus pluvialis. J. Ind. Microbiol. Biotechnol. 23:691-696.

Zhekisheva, M., Boussiba, S., Khozin-Goldberg, I., Zarka, A. \& Cohen, Z. 2002. Accumulation of oleic acid in Haematococcus pluvialis (Chlorophyceae) under nitrogen starvation or high light is correlated with that of astaxanthin esters. J. Phycol. 38:325-331.

Zhong, Y. -J., Huang, J. -C., Liu, J., Li, Y., Jiang, Y., Xu, Z. -F., Sandmann, G. \& Chen, F. 2011. Functional characterization of various algal carotenoid ketolases reveals that ketolating zeaxanthin efficiently is essential for high production of astaxanthin in transgenic Arabidopsis. J. Exp. Bot. 62:3659-3669.

Zlotnik, I., Sukenik, A. \& Dubinsky, Z. 1993. Physiological and photosynthetic changes during the formation of red aplanospores in the Chlorophyte Haematococcus pluvialis. J. Phycol. 29:463-469. 